\title{
Utilización de ibuprofeno por los usuarios de la farmacia comunitaria
}

\author{
Esther Garrigós Timoneda \\ Graduada en Farmacia en la Universidad de Barcelona. Farmacéutica comunitaria en Viladecans (Barcelona).
}

\section{PALABRAS CLAVE}

Ibuprofeno, dosis, indicaciones, eventos cardiovasculares, seguridad, farmacia comunitaria

\section{ABREVIATURAS}

AEMPS: Agencia

Española de

Medicamentos y

Productos Sanitarios

BIFAP: Base de datos

para la Investigación

Farmacoepidemiológica

en Atención Primaria

COX: ciclooxigenasa

EMA: European

Medicine Agency

(Agencia Europea del

Medicamento)

ISRS: inhibidores

selectivos de la

recaptación de la

serotonina

PRAC: Comité de

Evaluación de Riesgos

en Farmacovigilancia

\section{KEYWORDS}

Ibuprofen, dose, indications, cardiovascular events, safety, community pharmacy

\section{RESUMEN}

Introducción: Ibuprofeno es un antiinflamatorio no esteroideo con propiedades antiinflamatorias, analgésicas y antipiréticas. La seguridad de ibuprofeno fue revisada en 2006 y finalmente en 2015 se confirmó que la administración de dosis altas de ibuprofeno se asocia a un mayor riesgo de trombosis arterial.

Objetivos: Comprobar la utilización de ibuprofeno por los usuarios de la farmacia comunitaria.

Material y métodos: Los datos de este estudio descriptivo, observacional y transversal se obtienen del cuestionario realizado a los clientes habituales de una farmacia de Barcelona, previamente firmando una hoja de consentimiento y finalmente dando consejos mediante un tríptico informativo.

Resultados: Se incluyeron 40 personas que superaban los criterios de inclusión, de las que sólo una persona tenía ibuprofeno como tratamiento crónico. El 82,5\% utilizaba la dosis de $600 \mathrm{mg}$ y un 15,0\% la de $400 \mathrm{mg}$ con una posología predominante, en ambos casos, de cada 8 horas. Un 55,0\% del total utilizaba ibuprofeno hasta que remitía el dolor y los usos resultaron variados, quedando en primera posición el dolor de cabeza.

Discusión: No se puede corroborar qué dosis es la más predominante en otros países, pero $\mathrm{s}$ podemos aclarar que en algunos, como es el caso de Francia, la dosis de $600 \mathrm{mg}$ no está comercializada. Esta resulta ser la dosis más usada entre los encuestados superando la dosis máxima de $1.200 \mathrm{mg}$ al día recomendada para evitar un posible riesgo cardiovascular. Por otra parte, todas las indicaciones del ibuprofeno que usan los encuestados son correctas, con excepción de la del resfriado que en España no está admitida.

\section{Using ibuprofen among users of community pharmacies}

\section{ABSTRACT}

Introduction: Ibuprofen is a non-steroidal anti-inflammatory with anti-inflammatory, analgesic and antipyretic properties. The safety of ibuprofen was revised in 2006 and finally in 2015 it was confirmed that administration of high doses of ibuprofen is associated with an increased risk of arterial thrombosis.

Objectives: Testing the use of ibuprofen by users of the community pharmacy.

Material and methods: Data from this study descriptive, observational and cross-sectional are obtained from questionnaire of regular customers of the pharmacy in particular before signing a consent form and then being given advice through an information leaflet.

Results: 40 people were included exceeding the inclusion criteria, of which only one person had ibuprofen as chronic treatment. 82.5\% used doses of $600 \mathrm{mg}$ and 15.0\% of $400 \mathrm{mg}$ with a predominant dosage, every 8 hours, in both cases. 55.0\% of people used ibuprofen until the pain subsided and uses were varied, being headache in first position.

Discussion: No one can corroborate what dose is most prevalent in other countries, but we can clarify that the dose of $600 \mathrm{mg}$ is not marketed in some of them, such as France. This turns out to be the most used doses among the survey respondents exceeding the maximum dose of $1200 \mathrm{mg}$ per day recommended by AEMPS to avoid a possible cardiovascular risk. Moreover, all ibuprofen indications that respondents used are correct, except of the cold that in Spain is not supported. Compared to other countries, we can see that this use is accepted in the United States and the United Kingdom by the relevant agencies of medication. 


\section{Introducción}

Ibuprofeno es un antiinflamatorio no esteroideo derivado del ácido propiónico con propiedades antiinflamatorias, analgésicas y antipiréticas [1]. Su mecanismo de acción es debido a la inhibición de la sintesis de prostaglandinas mediante la inhibición de la ciclooxigenasa (COX-1 y COX-2). Las prostaglandinas desempeñan un papel esencial en la aparición de la fiebre, el dolor y la inflamación [1,2].

Fue descubierto por Stewart Adams junto con John Nicholson y Colin Burrows en 1961. El objetivo era encontrar fármacos con una mayor eficacia que Aspirina ${ }^{\circledR}$ y los corticoides para tratar la artritis reumatoide [2]. Hoy en día se trata de un medicamento mundialmente conocido, que forma parte de los medicamentos indispensables en la lista de la Organización Mundial de la Salud [3]. Por ello y porque resulta ser un medicamento muy económico se trata de uno de los fármacos más dispensados en las farmacias [4].

Después de que en 2004 rofecobix, inhibidor selectivo de la COX-2, fuera retirado del mercado por riesgo cardiovascular, se empezó a estudiar este posible efecto adverso en los medicamentos que inhiben tanto la COX-1 como la COX-2 [5]. Al revisar la seguridad de ibuprofeno en 2006 se confirmó una posible relación del consumo de este fármaco con un incremento del riesgo cardiovascular de tipo aterotrombótico, principalmente infarto de miocardio, ictus y problemas vasculares arteriales periféricos [6,7].

Posteriormente se realizaron $\mathrm{mu}-$ chos estudios para aclarar el nivel de riesgo cardiovascular y en 2012 se corroboró que la administración de dosis iguales o inferiores a $1.200 \mathrm{mg} /$ día de ibuprofeno parecía más seguro que el uso de dosis mayores $[6,8]$.

Finalmente, en 2015 se publicó una alerta en la Agencia Española de Medicamentos y Productos Sanitarios (AEMPS) que confirmó que la administración de dosis altas de ibuprofeno (iguales o mayores de $2.400 \mathrm{mg} /$ día) se asocia a un mayor riesgo de trombosis arterial [9], recomendando su administración a dosis menores.

El Comité de Evaluación de Riesgos en Farmacovigilancia (PRAC) de la Agencia Europea del Medicamento (EMA) afirma que faltan datos sobre los riesgos trombóticos que causa el ibuprofeno a dosis de 1.200 a 2.400 mg al día. Por lo tanto, hoy en día, todavía no se puede determinar exactamente cómo varía el riesgo en este intervalo posológico [10].

\section{Objetivos}

El objetivo principal de este trabajo es conocer las características de la utilización de ibuprofeno por los usuarios de la farmacia comunitaria.

Como objetivos específicos se planteó estudiar los siguientes aspectos referentes a la utilización de ibuprofeno:

- Dosis e indicación.

- Pauta.

- Duración del tratamiento.

- Efectos adversos e interacciones.

\section{Material y métodos}

La primera parte del trabajo se ha basado en la búsqueda de artículos científicos y metaanálisis sobre la seguridad y efectividad del ibuprofeno y su utilización en el ámbito ambulatorio. Por otra parte, a través de las fichas técnicas de la AEMPS, se han revisado las interacciones de ibuprofeno con los medicamentos crónicos de los pacientes encuestados [1].

Tipo de estudio,

ámbito local y temporal

Estudio descriptivo, observacional y transversal realizado en una farmacia comunitaria de la ciudad de Barcelona. La duración del estudio ha sido de cuatro meses, de noviembre de 2015 a febrero de 2016.

En primer lugar se hizo una búsqueda en el programa informático de gestión farmacéutica del que dispone la farmacia. A través del apartado de estadísticas de venta y compra se pudo constatar el número total de envases dispensados durante el año 2015 con el principio activo incluido en el estudio (ibuprofeno) y la relación de pacientes con historial farmacoterapéutico en la farmacia que lo utilizaban.

\section{Sujetos}

Criterios de inclusión: pacientes habituales de la farmacia, mayores de edad, que durante el período del estudio acudieron a recoger su medicación, aunque no fuera ibuprofeno, pero que lo utilizaban habitualmente, tanto de $600 \mathrm{mg}$ como de $400 \mathrm{mg}$ en comprimidos y sobres.

Criterios de exclusión: menores de edad, personas mayores con reducida comprensión de los fármacos que toman, embarazadas, ibuprofeno administrado por vía intravenosa o tópica, personas que no tomen este fármaco.

La selección de las personas se basó en escoger a los clientes habituales de la farmacia para conocer los medicamentos de uso habitual junto con ibuprofeno. No se esperó a que solicitasen el medicamento sino que se realizaron las encuestas a los clientes habituales de la farmacia que lo tenían en su historial farmacoterapéutico.

A los que accedieron a realizar el estudio y superaron los criterios de inclusión, se les hizo una entrevista semiestructurada que recogía los siguientes datos: dosis, posología, patología tratada y observaciones del ibuprofeno, además del tratamiento crónico usado durante la administración de este antiinflamatorio.

Para mostrar su conformidad en formar parte del estudio todos los sujetos cumplimentaron una hoja de consentimiento. Al finalizar las distintas preguntas, se les dio información escrita en formato tríptico y diferentes recomendaciones dependiendo de las respuestas proporcionadas en la encuesta.

\section{Resultados}

De los 52 pacientes que aceptaron participar en el estudio, 12 (23,1\%) fueron excluidos porque ya no tomaban ibuprofeno en el momento de la encuesta por diferentes motivos: 4 por alergias, 3 por interacciones con su tratamiento crónico y 5 por no necesidad. Completaron el estudio 40 personas, 21 (52,2\%) mujeres y 19 (47,5\%) hombres, cuya distribución por edad y sexo se muestra en la tabla 1 .

Tabla 1 Caracteristicas de la muestra en cuanto a edad y sexo

\begin{tabular}{|l|c|c|c|}
\hline Edad/sexo & Mujeres & Hombres & Total \\
\hline 18-35 años & 5 & 3 & 8 \\
\hline 36-64 años & 12 & 5 & 17 \\
\hline$>64$ años & 4 & 11 & 15 \\
\hline Total & 21 & 19 & 40 \\
\hline
\end{tabular}




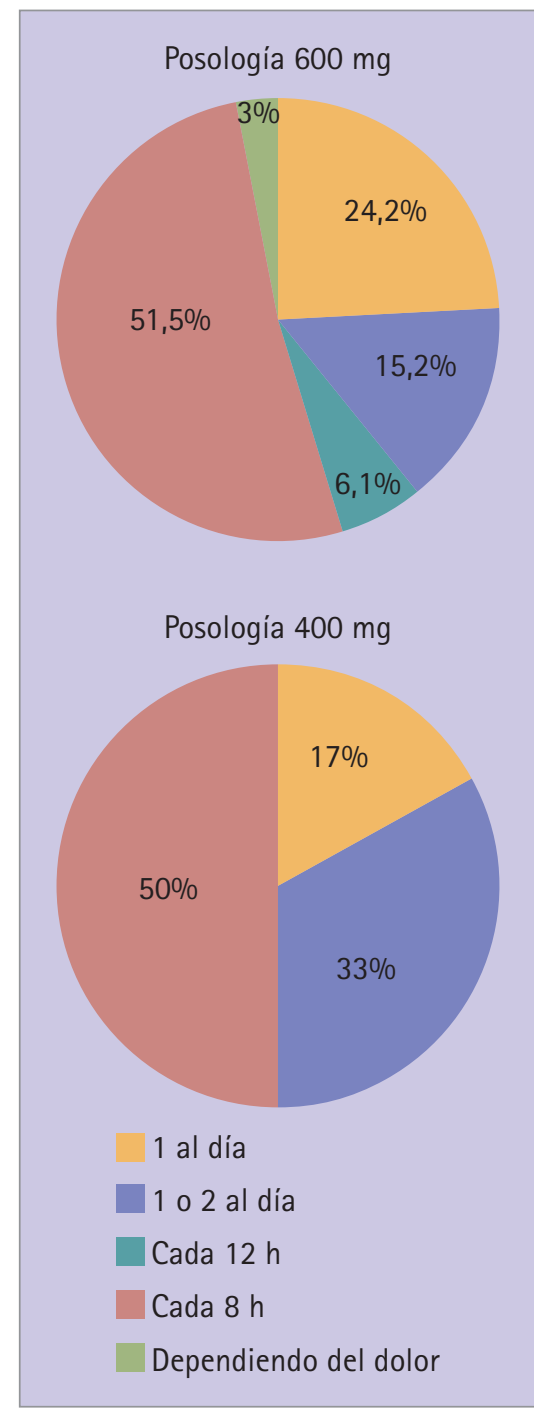

Figura 1 Pautas posológicas de las dosis de ibuprofeno de 600 y $400 \mathrm{mg}$

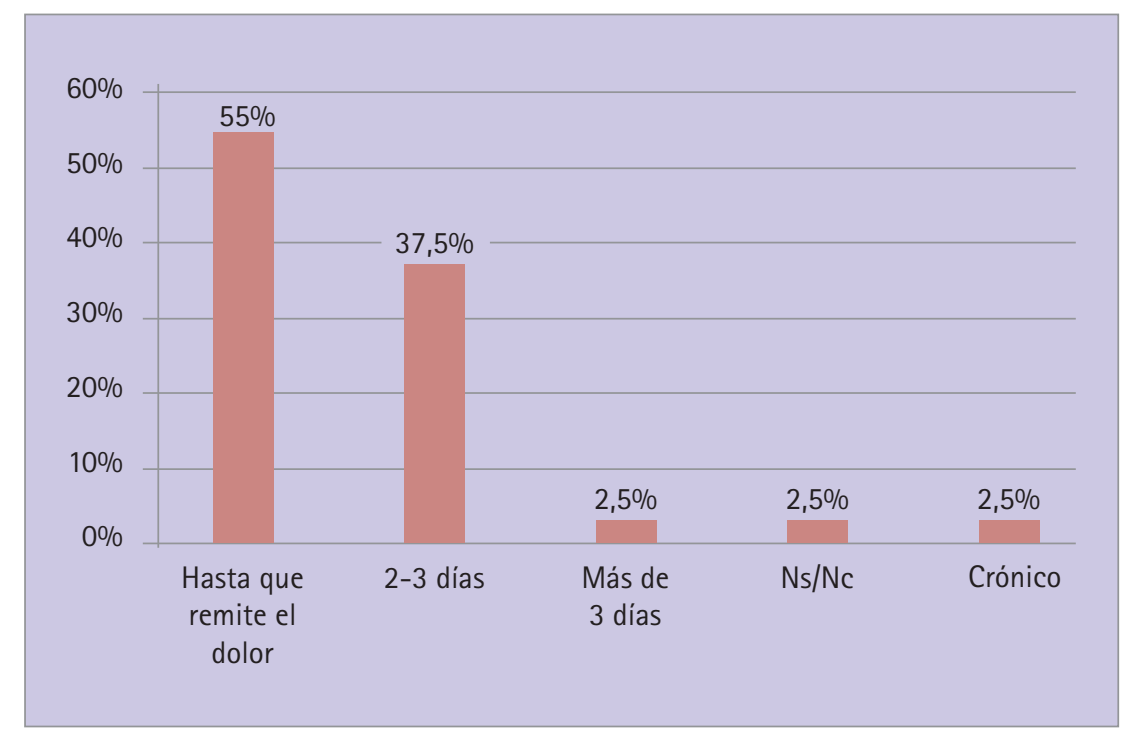

Figura 2 Duración de la administración de ibuprofeno

De los encuestados, $33(82,5 \%)$ tomaban ibuprofeno de $600 \mathrm{mg}, 6$ $(15,0 \%)$ de $400 \mathrm{mg}$ y $1(2,5 \%)$ no sabía qué dosis solía tomar. Las diversas posologías usadas y la duración de los tratamientos se presentan en las figuras 1 y 2 .

Los problemas de salud para los cuales los pacientes afirmaron utilizar ibuprofeno se muestran en la figura 3. En el caso de dismenorrea, se debe tener en cuenta que la edad media de la menopausia es a los 50 años [11], por tanto, con este dato, se pueden excluir las mujeres de 50 años o más y quedan 6 mujeres de un total de 21. De estas, 6,2 (33\%) utilizaban ibuprofeno para dolores menstruales.

21 personas $(52,5 \%)$ toman tratamiento crónico al mismo tiempo que usan el ibuprofeno, hay más hombres $(63,0 \%)$ con tratamiento crónico que mujeres $(43,0 \%)$ y la mayoría son del rango de edad de más de 64 años. 15 personas (71,0\% de los que toman otros tratamientos al mismo tiempo que ibuprofeno) presentan interacciones con ibuprofeno (figura 4).

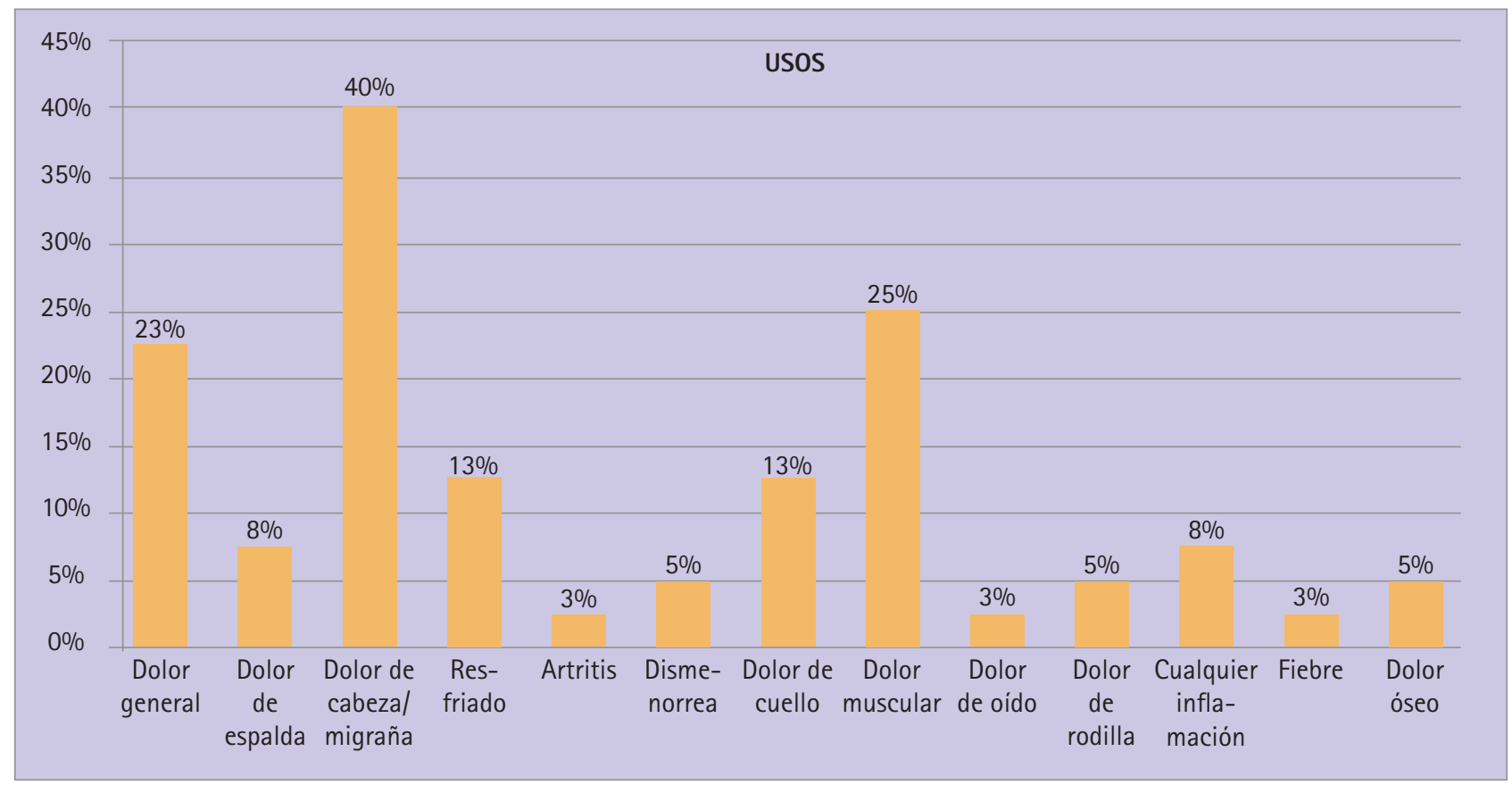

Figura 3 Problemas de salud para los cuales los pacientes encuestados toman ibuprofeno 


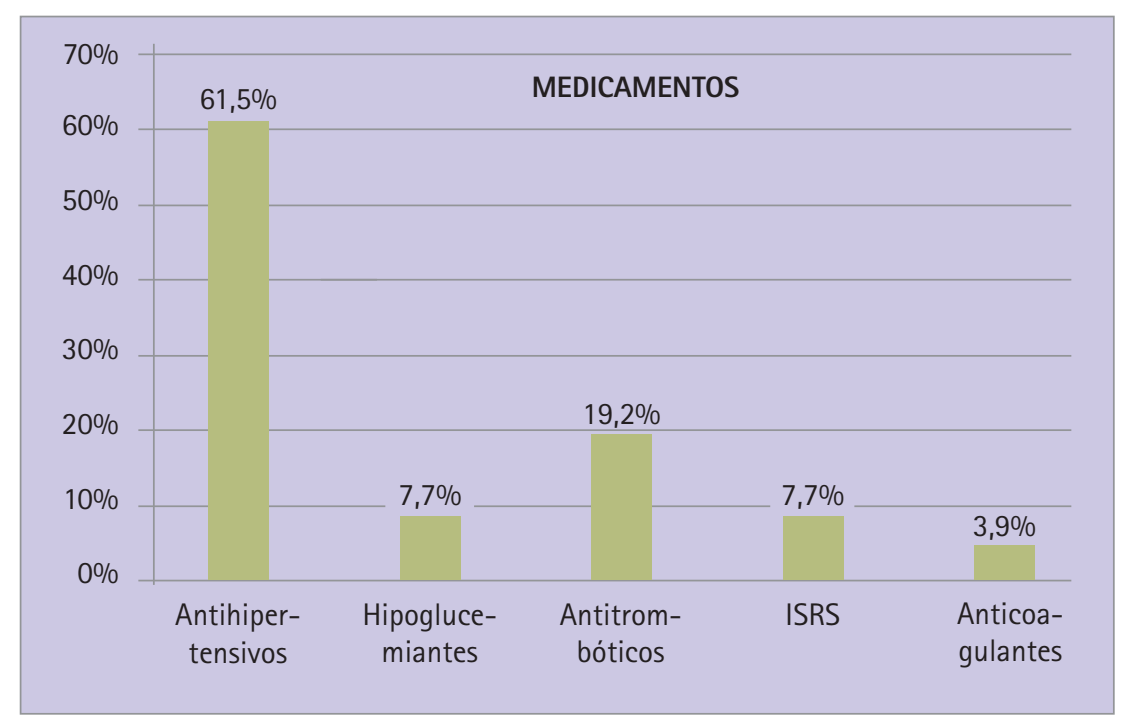

Figura 4 Tipos de medicamentos que interaccionan con ibuprofeno y porcentaje de pacientes que lo utilizan

\section{Discusión}

Las limitaciones más relevantes del estudio son, por un lado la pequeña muestra encuestada, por lo que los resultados no son significativos y que se basa en una encuesta realizada a pacientes, alguno de los cuales solicitaba el medicamento en ese momento y por lo tanto es posible que las respuestas no sean totalmente adecuadas a la realidad del uso que hacen del medicamento.

Respecto a la dosis, se observa que una gran mayoría $(82,5 \%)$ de las personas entrevistadas toman ibuprofeno de $600 \mathrm{mg}$. Los principales motivos son:

- El médico receta esta dosis.

- El desconocimiento de la existencia de la forma farmacéutica de ibuprofeno $400 \mathrm{mg}$.

- El farmacéutico dispensa el formato de $600 \mathrm{mg}$ por defecto.

- Por costumbre.

No se pudo comparar si esta situación se daba en otros países por la poca o nula existencia de este tipo de estudios. Sin embargo, tras buscar en las agencias del medicamento de diferentes países: España, Reino Unido, Alemania, Francia y Estados Unidos, se ha comprobado que Francia es el único país de estos cinco que no tiene comercializado la presentación de ibuprofeno de $600 \mathrm{mg}$ [12].

La posología predominante ha sido de un comprimido/sobre cada 8 horas, tanto en la dosis de $400 \mathrm{mg}$ como la de $600 \mathrm{mg}$. Por lo tanto, los que usan la dosis de $600 \mathrm{mg}$ supera- rían la cantidad máxima diaria recomendada de $1.200 \mathrm{mg}$ al día.

Respecto a la duración un 71,0\% de los que toman ibuprofeno de $600 \mathrm{mg}$ cada 8 horas siguen el tratamiento hasta que remite el dolor.

La seguridad del ibuprofeno se basa en la administración de menos de $1.200 \mathrm{mg}$ al día durante 7-10 días $[2,9]$. Diferentes estudios europeos constatan que si se supera esta dosis y/o se administra ibuprofeno a largo plazo puede provocar problemas cardiovasculares graves $[4,5,13,14]$. Por lo tanto, este $71 \%$ que está ya superando la dosis diaria recomendada también podría estar superando la duración aconsejada.

En cuanto a los usos del ibuprofeno, tanto en España como en la mayoría de países europeos su uso es muy similar. Todos los usos que mencionan los encuestados son correctos según la ficha técnica del ibuprofeno [1] a excepción de uno, el resfriado, que no está aprobado en España. En cambio, si se revisan las fichas técnicas de la Agencia Reguladora de Medicamentos y Productos Sanitarios del Reino Unido [15] o de la Biblioteca Nacional de Estados Unidos de Medicina [16] estos afirman que es correcto este uso terapéutico. Cinco de los usuarios estudiados confirman que se toman el antiinflamatorio para el resfriado y que los síntomas alivian y/o desaparecen a los pocos días. Los metaanálisis muestran que hay poca información sobre la efectividad de ibuprofeno en gripe y resfriados [17]. Sin embargo, sí que se comprueba la reducción significativa de diferentes síntomas de la gripe al administrar este antiinflamatorio. Ibuprofeno disminuye la fiebre, el dolor de cabeza, dolor de oídos, dolor muscular, articular y estornudos, pero no se ha demostrado que acorte la duración de la gripe $[18,19]$.

Hay un total de 15 personas con interacciones de su medicación crónica con ibuprofeno, que no deberían tomar esta medicación si no es bajo supervisión médica y todos confirmaron que no notifican a sus médicos cuando se administran el ibuprofeno. Además de que ya corren un riesgo por el hecho de tomar este tipo de antiinflamatorio, hay dos personas que superan la dosis recomendada al día, lo que provoca que se encuentren en mayor riesgo de sufrir un problema cardiovascular.

A la vista de los resultados de este trabajo, y a pesar de sus limitaciones, es evidente que todos los profesionales sanitarios debemos transmitir a los pacientes un mismo mensaje indicándoles la dosis más segura de ibuprofeno en cada caso e intentando divulgar poco a poco a la sociedad el uso y la posología correctos de este fármaco.

\section{Agradecimientos}

En primer lugar al titular de la Farmacia Santiago Gómez donde he realizado las encuestas, a los trabajadores y sobre todo, a los pacientes, por su amabilidad y la ayuda que me han facilitado para que este proyecto se pudiera llevar a cabo. En segundo lugar, quiero dar las gracias a mi tutora de la Facultad de Farmacia, Neus Caelles, por sus indicaciones y por proporcionarme información muy útil. Y, por último, agradecer el apoyo que he recibido durante todos estos meses de trabajo por parte de mi familia y pareja.

\section{Referencias bibliográficas}

1. Agencia Española de Medicamentos y Productos Sanitarios (AEMPS) [Internet]. Centro de Información online de Medicamentos de la AEMPS (CIMA). Ficha técnica Ibuprofeno Pensa. Diciembre 2015. [Acceso 2/12/2015]. Disponible en: http://www.aemps. gob.es/cima/pdfs/es/ft/62973/ FT_62973.pdf

2. Rainsford K.D. Ibuprofen: Discovery, Development and Therapeutics [Libro en Internet] $1^{\text {a }}$ ed. Reino Unido: 
John Wiley \& Sons; 2015. p. 1-19. [Acceso 4/12/2015]. Disponible en: http://www.vixri.ru/d3/K.D.Rainsford\%20-\%20Ibuprofen\%20Pharmacology, $\% 20$ Therapeutics\%20and $\% 20$ Side\%20Effects\%20-\%202012.pdf

3. World Health Organization [Internet]. WHO Model List of Essential Medicines - 18th List (April 2015). [Acceso 15/12/2015]. Disponible en: http://www.who.int/medicines/ publications/essentialmedicines/ EML2015_8-May-15.pdf

4. Ministerio de Sanidad Servicios Sociales e Igualdad. Informe anual del Sistema Nacional de Salud 2013. [Acceso 15/12/2015]. Disponible en: http://www.msssi.gob.es/organizacion/sns/planCalidadSNS/pdf/ equidad/Informeanual2013/Informe_2013_SNS_WEB.pdf

5. Trelle S, Reichenbach S, Wandel S, Hildebrand P, Tschannen B, Villiger PM, et al Cardiovascular safety of non-steroidal anti-inflammatory drugs: network meta-analysis. BMJ [online]. 2011; 342: c7086 doi:10.1136/bmj.c7086

6. Agencia Española de Medicamentos y Productos Sanitarios [Internet]. Seguridad cardiovascular de los AINE tradicionales: conclusiones de la revisión de los últimos estudios publicados. 2012. [Acceso 2/2/2016]. Disponible en: http://www.aemps.gob.es/ informa/notasInformativas/medicamentosUsoHumano/seguridad/2012/ docs/NI-MUH_FV_15-2012.pdf

7. Agencia Española de Medicamentos y Productos Sanitarios [Internet]. Comunicación sobre riesgos de medicamentos para profesionales sanitarios. Actualización sobre los riesgos de tipo aterotrombótico de los COXIBs y AINE tradicionales. Nota informativa 26/10/2006. [Acceso 20/1/2016]. Disponible en: http://www.aemps.gob.es/ informa/notasInformativas/medicamentosUsoHumano/seguridad/2006/ docs/NI_2006-10_coxibs_AINE.pdf

8. McGettigan P, Henry D. Cardiovascular risk with non-steroidal anti-inflammatory drugs: Systematic review of population-based controlled observational studies. PLoS Med [Internet]. 2011; 8(9): e1001098. doi:10.1371/ journal.pmed.1001098

9. Agencia Española de Medicamentos y Productos Sanitarios. [Internet]. Riesgo cardiovascular de dosis altas de ibuprofeno o dexibuprofeno: recomendaciones de uso. Recomendaciones del Comité Europeo para la Evaluación de Riesgos en Farmacovigilancia (PRAC) 12/04/2015. [Acceso 2/12/2015]. Disponible en: http://www.aemps.gob.es/informa/ notasInformativas/medicamentosUsoHumano/seguridad/2015/docs/NIMUH_FV_04-ibuprofeno-dexibuprofeno.pdf

10. Agencia Europea del Medicamento [Internet]. Anexo II Conclusiones científicas y motivos para la modificación de las condiciones de las autorizaciones de comercialización. Conclusiones científicas. [Acceso 2/2/2016]. Disponible en: http:// www.ema.europa.eu/docs/es_ES/ document_library/Referrals_document/Ibuprofen_and_dexibuprofen_31/Position_provided_by_CMDh/ WC500189664.pdf

11. MedlinePlus. Biblioteca Nacional de Medicina de los EE.UU. Menopausia. [Internet]. 2016. [Acceso 15/2/2016]. Disponible en: https://www.nlm.nih. gov/medlineplus/spanish/ency/article/000894.htm

12. Agence Nationale de Sécurité du Médicament et des Produits de Santé [Internet]. Répertoire des Spécialités Pharmaceutiques. Substance active 'ibuprofène' commercialisée. Feb. 2016. [Acceso 18/2/2016]. Disponible en: http://agence-prd.ansm.sante.fr/ php/ecodex/index.php\#result

13. Medicines and Healthcare products Regulatory Agency [Internet]. Cox-2 selective inhibitors and non-steroidal anti-inflammatory drugs (NSAIDs): Cardiovascular safety. Enero 2015 [Acceso 15/1/2016]. Disponible en: https://www.gov.uk/government/ publications/cox-2-selective-inhibitors-and-non-steroidal-anti-inflam- matory-drugs-nsaids-cardiovascular-safety

14. Medicines and Healthcare products Regulatory Agency [Internet]. MHRA public assessment report. Non-steroidal anti-inflammatory drugs and cardiovascular risks in the general population. Enero 2010. [Acceso 15/1/2016]. Disponible en: http:// webarchive.nationalarchives.gov. uk/20141205150130/http://www. mhra.gov.uk/home/groups/pl-p/documents/websiteresources/con068576. pdf

15. Medicines \& Healthcare products Regulatory Agency [Internet]. Regulating Medicines and Medical Devices. Patient information leaflet. May. 2016. [Acceso 26/03/17]. Disponible en: http://www.mhra.gov. uk/home/groups/spcpil/documents/ spcpil/con1490329570039.pdf

16. MedlinePlus. Biblioteca Nacional de Medicina de los EE.UU [Internet]. Ibuprofen. Set. 2015. [Acceso 18/2/2016]. Disponible en: https: www.nlm.nih. gov/medlineplus/druginfo/meds/ a682159.html\#side-effects

17. Eccles R. Efficacy and safety of over-the-counter analgesics in the treatment of common cold and flu. J Clin Pharm Ther. [Internet]. 2006 [Acceso 17/12/2015]. Disponible en: https://www.ncbi.nlm.nih.gov/pubmed/16882099

18. Grebe W, Ionescu E, Gold M, Liu J, Frank W. A multicenter, randomized, double-blind, double-dummy, placebo- and active-controlled, parallel-group comparison of diclofenac-K and ibuprofen for the treatment of adults with influenza-like symptoms. Clinical Therapeutics [Internet]. 2003; (2). [Acceso 17/12/2015]. Disponible en: https://www.ncbi.nlm.nih.gov/ labs/articles/12749506/

19. Winther B, Mygind N. The therapeutic effectiveness of ibuprofen on the symptoms of naturally acquired common colds. Am J Rhinol. [Internet]. Julio 2001. [Acceso 17/12/2015]. Disponible en: https://www.ncbi.nlm. nih.gov/pubmed/11554655 